\title{
PINK1 expression increases during brain development and stem cell differentiation, and affects the development of GFAP- positive astrocytes
}

Insup Choi ${ }^{1,2}$, Dong-Joo Choi ${ }^{1}$, Haijie Yang ${ }^{3}$, Joo Hong Woo ${ }^{2}$, Mi-Yoon Chang ${ }^{4}$, Joo Yeon Kim ${ }^{5}$, Woong Sun ${ }^{5}$, Sang-Myun Park ${ }^{1,2,3}$, Ilo Jou ${ }^{1,2,3}$, Sang-Hun Lee ${ }^{4}$ and Eun-Hye Joe $e^{1,2,3,6,7^{*}}$

\begin{abstract}
Background: Mutation of PTEN-induced putative kinase 1 (PINK1) causes autosomal recessive early-onset Parkinson's disease (PD). Despite of its ubiquitous expression in brain, its roles in non-neuronal cells such as neural stem cells (NSCs) and astrocytes were poorly unknown.

Results: We show that PINK1 expression increases from embryonic day 12 to postnatal day 1 in mice, which represents the main period of brain development. PINK1 expression also increases during neural stem cell (NSC) differentiation. Interestingly, expression of GFAP (a marker of astrocytes) was lower in PINK1 knockout (KO) mouse brain lysates compared to wild-type (WT) lysates at postnatal days 1-8, whereas there was little difference in the expression of markers for other brain cell types (e.g., neurons and oligodendrocytes). Further experiments showed that PINK1-KO NSCs were defective in their differentiation to astrocytes, producing fewer GFAP-positive cells compared to WT NSCs. However, the KO and WT NSCs did not differ in their self-renewal capabilities or ability to differentiate to neurons and oligodendrocytes. Interestingly, during differentiation of KO NSCs there were no defects in mitochondrial function, and there were not changes in signaling molecules such as SMAD1/5/8, STAT3, and HES1 involved in differentiation of NSCs into astrocytes. In brain sections, GFAP-positive astrocytes were more sparsely distributed in the corpus callosum and substantia nigra of KO animals compared with WT.
\end{abstract}

Conclusion: Our study suggests that PINK1 deficiency causes defects in GFAP-positive astrogliogenesis during brain development and NSC differentiation, which may be a factor to increase risk for PD.

Keywords: PINK1, Neural stem cell, Astrocyte, Parkinson's disease

\section{Background}

PTEN-induced putative kinase 1 (PINK1) is a PDrelated gene whose mutation causes an autosomal recessive early-onset PD [1]. PINK1 plays diverse roles. For example, it regulates mitochondrial function [2], which is linked to ATP generation, oxygen consumption [3-5], and ROS production [6]. In addition, PINK1 regulates the AKT-mTOR and HIF-1 alpha pathways to mediate

\footnotetext{
* Correspondence: ehjoe@ajou.ac.kr

${ }^{1}$ Neuroscience Graduate Program Department of Biomedical Sciences, Ajou University School of Medicine, Suwon, Korea

${ }^{2}$ Chronic Inflammatory Disease Research Center, Ajou University School of Medicine, Suwon, Korea

Full list of author information is available at the end of the article
}

proliferation, survival, metabolism, and inflammation etc. [7-12]. Finally, PINK1 deficiency reportedly reduces astrocyte proliferation [7] and neurite outgrowth [13], suggesting that this deficiency may affect brain development and/or injury repair.

Astrocytes, which are the most abundant cells in the brain, express glial fibrillary acidic protein (GFAP) and are known to play important roles in developing, intact, and injured brains. Astrocytes regulate synaptogenesis [14], neural activity, and neural circuit formation in both developing and injured brains [15-17]. In intact brain, astrocytes support neurons by providing nutrients and growth factors [18-20], and maintaining the homeostasis 
of extracellular potassium and glutamate [21, 22]. In injured brain, astrocytes become hypertrophic, exhibit increased GFAP expression, and proliferate, thereby isolating injury sites, preventing oxidative stress and neuronal death, and decreasing inflammation [23-28]. The neural stem cells (NSCs) in the subventricular zone (SVZ) of the brain are a specialized form of GFAP-expressing astrocytes [29] that contributes to injury repair. In ischemic brain, it was recently reported that astrocytes differentiate into new neurons and participate in regenerating the injured brain [30]. Therefore, defects of astrogliogenesis could cause brain abnormalities, including neurodegeneration [31].

In this study, we show that PINK1 expression increases during brain development and NSC differentiation, whereas PINK1 deficiency decreases GFAP expression during these processes. Subsequent experiments revealed that PINK1 deficiency causes defects in astrogliogenesis, decreasing the number of GFAP-positive astrocytes and causing abnormalities in their locations and configurations in the corpus callosum, and substantia nigra reticulate. Collectively, these findings suggest that defects in GFAP-positive astrogliogenesis could be a mechanism through which PINK1 deficiency could contributes to the development of PD.

\section{Results}

The expression levels of PINK1 increase during brain development, and GFAP expression is attenuated in PINK1-deficient mouse brains

Since PINK1 is closely associated with the signaling pathways that regulate cell proliferation, survival, and differentiation [7-9], we first examined the expression levels of PINK1 during brain development during a period characterized by the vigorous proliferation and differentiation of brain cells. Brain lysates were prepared from samples taken on embryonic day 11.5 (E11.5) through E17.5, as well as on postnatal day 1 (P1), P7, and at 8 weeks after birth. The protein expression of the neuronal marker, TUJ-1, gradually increased from E11.5 to adulthood and, as previously reported [32], the astrocyte marker, GFAP, appeared at around P1 (Fig. 1a). The oligodendrocyte marker, myelin basic protein (MBP), was not detected up to P7, but could be detected at 8 weeks (Fig. 1a). Interestingly, the PINK1 protein expression levels showed some correlation with brain development, increasing from E11.5 to a peak at E17.5 and P1, and then decreasing at P7 and 8 weeks (Fig. 1a). The mRNA levels of PINK1 showed a similar expression pattern, gradually increasing from E11.5 to a peak at $\mathrm{P} 1$, and then decreasing at $\mathrm{P} 7$ and 8 weeks (Fig. 1b). We also found that protein expression of Parkin, another PD gene [33], showed similar patterns to that of PINK1 (Additional file 1: Figure S1).
Since PINK1 expression was upregulated during brain development, particularly during the period when the expression levels of TUJ1 and GFAP were also increased (Fig. 1a, b), we questioned whether PINK1 could be functionally associated with the expression levels of TUJ1 and/or GFAP. Accordingly, we compared the expression levels of GFAP, TUJ1, and MAP2 (another marker of neurons) in WT and PINK1-knockout (KO) brains at P1, P8, and 8 weeks. Using Western blot, we confirmed absence of PINK1 protein expression in PINK1 KO mice (Additional file 2: Figure S2). The PINK1 deficiency in PINK1-KO mice was usually confirmed using genotyping prior to the preparation of brain lysates as previously described [34]. Interestingly, we found that GFAP protein levels in PINK1-KO brain were lower than in WT brain at P1 and P8, whereas there was little difference in the levels of TUJ1 and MAP2 (Fig. 1c). At 8 weeks, however, there was no significant difference in the levels of GFAP as well as TUJ1 or MAP2 in WT and PINK1-KO brains (Fig. 1c). These results suggest that PINK1 regulates brain development, particularly, GFAP expression.

\section{The expression levels of PINK1 increase during NSC differentiation, and GFAP expression is attenuated in PINK1-deficient NSCs in vitro}

Since GFAP expression differed in WT and KO during brain development (Fig. 1c), we examined whether PINK1 regulates the proliferation and/or differentiation of NSCs obtained from E13.5 mouse brains. The NSCs were cultured as neurospheres, and their proliferative capacity was assessed by counting the number and size of secondary neurospheres, measuring $\left[{ }^{3} \mathrm{H}\right]$-thymidine incorporation, and assessing the cell numbers. We previously reported that PINK1 regulates astrocyte proliferation [7]. However, proliferation defect was not found in PINK1-KO NSCs since the number and size of neurospheres derived from WT and PINK1-KO NSCs were similar (Fig. 2a and b). Additionally, the cell numbers and $\left[{ }^{3} \mathrm{H}\right]$-thymidine incorporation level did not significantly differ between WT and PINK1-KO NSCs (Fig. 2c and $\mathrm{d}$ ), and the proliferation capacities of WT and PINK1-KO NSCs did not significantly differ even at a later passage (passage 8) (Fig. 2e).

We further examined whether PINK1 regulates NSC differentiation. Interestingly, the mRNA and protein levels of PINK1 significantly increased during differentiation into neurons and astrocytes, as demonstrated by increases in MAP2, TUJ1, and GFAP after 1-5 days of differentiation, and a decrease in nestin (an NSC marker) expression beginning after $1 \mathrm{~d}$ of differentiation (Fig. 3a, b). As in developing brain, Parkin protein expression also increased during differentiation of NSCs 


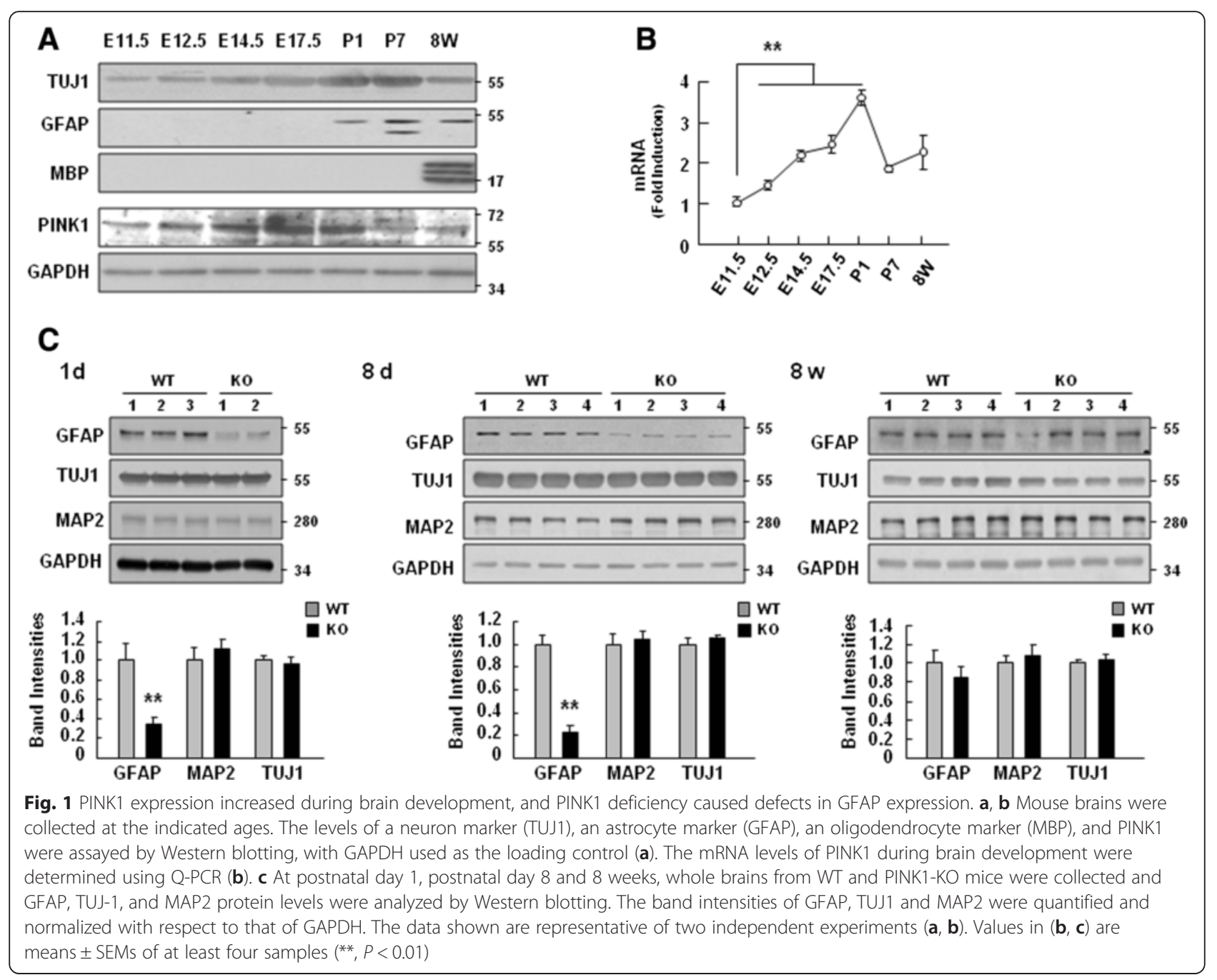

similar to PINK1 protein expression (Additional file 3: Figure S3).

We next compared the differentiation patterns of WT and PINK1-KO NSCs. During the induction of NSC differentiation, the protein levels of MAP2 and TUJ1 were similar in WT and PINK1-KO (Fig. 3c). However, GFAP protein levels were significantly lower in PINK-1 KO cells compared to WT NSCs on days 3 and 5 of differentiation (Fig. 3c). The decrease was not due to cell death, as indicated by similar levels of cleaved PARP, cleaved caspase-3, and LDH between WT and PINK1-KO NSCs (Additional file 4: Figure S4). We also examined differentiation of NSCs in the presence of CNTF, a well known strong inducer of astrocyte differentiation [35]. On day 5 of differentiation, CNTF dose-dependently (in the range of $0.1-1 \mathrm{ng} / \mathrm{ml}$ ) increased the differentiation of NSCs into astrocytes, as demonstrated by GFAP expression (Fig. 3d). Furthermore, the GFAP protein level was lower in PINK1-KO NSCs than in WT NSCs (Fig. 3d). Immunostaining with antibodies specific for GFAP, MAP2 and CNPase revealed that there were significantly fewer GFAP-positive cells among PINK1-KO NSCs compared to WT NSCs on day 5 of differentiation (17.7 \% vs. $6.8 \%)$, whereas the numbers of MAP2- (43.1\% vs. $42.4 \%)$ and CNPase-positive cells ( $4.8 \%$ vs. $4.3 \%$ ) were not significantly different (Fig. 3e). Collectively, these results suggest that PINK1 is required for the differentiation of NSCs into GFAP-positive astrocytes.

\section{Neither GFAP mRNA expression nor signaling pathways involved in gliogenesis are changed in PINK1-deficient NSCs}

In an effort to identify the mechanisms responsible for decreasing the differentiation of PINK1-KO NSCs into GFAP-positive astrocytes, we examined the activation levels of the signaling molecules involved in astrogliogenesis, including STAT3 [36-38], SMAD1/5/8 [39], and HES1 [40]. The activation of these molecules are evaluated by phosphorylation (SMAD1/5/8 and STAT3) [41, 42], or expression (HES1) [43]. Unexpectedly, however, 


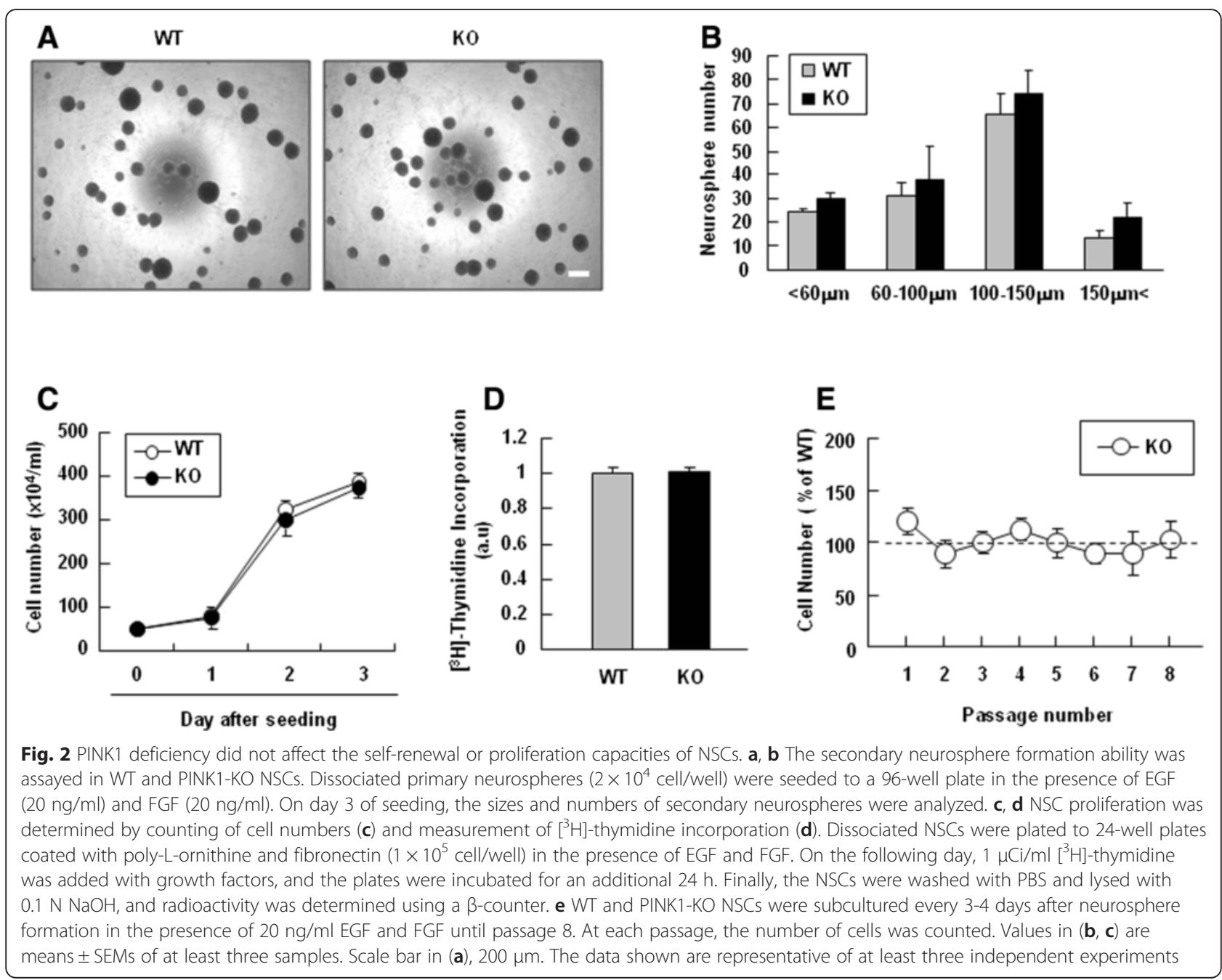

there was no difference in the levels of pSMAD1/5/8, pSTAT3, and HES1 (Fig. 4a, b), even in the presence of CNTF (Fig. 4d). Accordingly, mRNA levels of GFAP did not differ significantly in WT and PINK1-KO NSCs during differentiation in the absence (Fig. 4c) and presence of CNTF (Fig. 4e), suggesting that PINK1 dose not regulate GFAP expression at transcriptional level.

In further studies, we examined the effect of proteasome inhibitors, MG132 and lactacystin, on GFAP expression. However, these inhibitors also had little effect on GFAP expression (Additional file 5: Figure S5), suggesting that PINK1 did not alter the protein stability of GFAP. Therefore, further studies are required to assess how PINK1 regulates GFAP expression and/or the generation of GFAP-positive astrocytes.

\section{Mitochondrial defects were not found in PINK1 deficient NSCs during differentiation}

Next, we examined the possible involvement of mitochondrial dysfunction in abnormal astrogliogenesis in PINK1 deficient NSCs, since we and others have found that PINK1 deficiency causes mitochondrial dysfunctions in neurons and astrocytes $[2,7,44]$. However, mitochondrial dysfunction was not detectable in PINK1 KO NSCs for up to 5 days after the induction of differentiation. WT and KO NSCs did not significantly differ in their mitochondrial membrane potential or ROS production, as measured by FACS analysis using MitoTracker Red CMXRos and carboxyl- $\mathrm{H}_{2}$ DFFDA, respectively (Fig. 5a). In addition, the mitochondrial DNA copy number did not differ between WT and PINK1-KO NSCs (Fig. 5b). These findings suggest that PINK1 may not be required for normal mitochondrial function in NSCs differentiation.

Differences in the distribution of GFAP-positive cells in the lateral ventricle and/or substantia nigra (SN) of WT and PINK1-KO mice

Next, we analyzed GFAP-positive astrocytes in several regions of WT and PINK1-KO mouse brains, including the lateral ventricles (Fig. 6a, b, c) and SN, where dopaminergic neuronal processes and cell bodies locate (Fig. 6a, d, e). In the cortex of P8 mice, GFAP immunoreactivity 


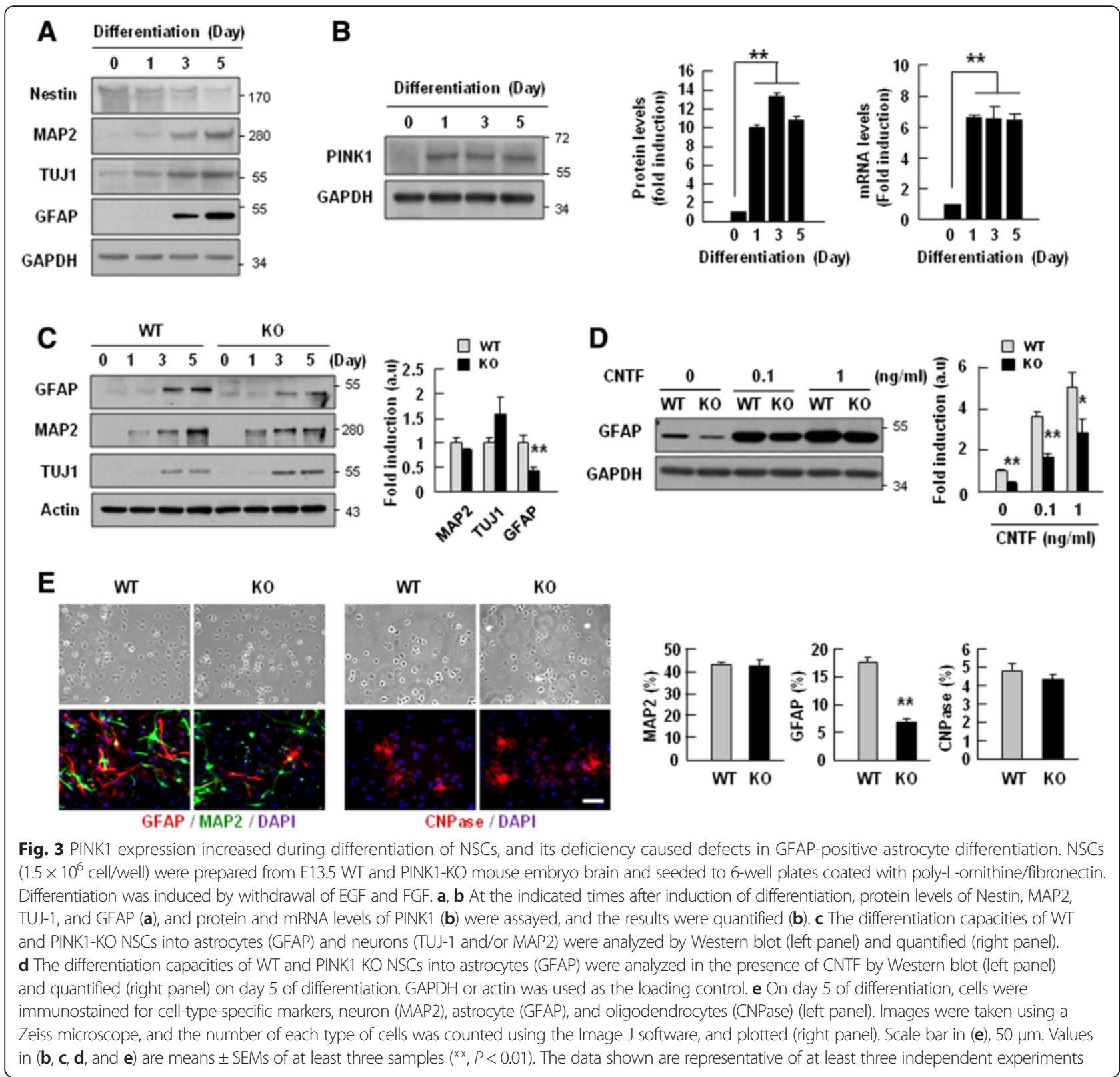

was detectable in the pia mater (arrowheads in Fig. 6b1 and $\mathrm{c} 1$ ) and the thin processes beneath this structure (arrows in Fig. $6 \mathrm{~b} 1$ and $\mathrm{c} 1$ ), but these processes were thicker and longer in PINK1-KO brains (arrows in Fig. 6b1 and c1). Interestingly, the morphology and/or distribution of GFAP-positive astrocytes in WT and KO mice differed in the corpus callosum (CC); in particular, the point at which the dorsal horn $(\mathrm{dh})$ of the lateral ventricle (which was not yet fully developed at this stage) connected to the CC was filled with GFAP-immunoreactive cells in WT but not in $\mathrm{KO}$ mice (arrows in Fig. 6b2 and c2). Finally, the P8 SN was densely populated with GFAP-immunoreactive astrocytes in WT brains, but only sparsely populated with these astrocytes in $\mathrm{KO}$ brains (arrows in Fig. $6 \mathrm{~d}$ and e). Image analysis using Image J (f) and western blot using brain lysates prepared from each brain regions (g) showed decrease in GFAP expression in PINK1 KO brain. Taken together, these results indicate that GFAP-positive astrocytes developed abnormally in PINK1-deficient mouse brains and NSCs.

\section{Discussion}

The results of this study show that PINK1 expression increases during brain development and NSC differentiation, and that this increase is related to changes in GFAP expression during these two processes. Furthermore, PINK1 deficiency decreased the differentiation of NSCs into GFAP-positive astrocytes, and caused defects 

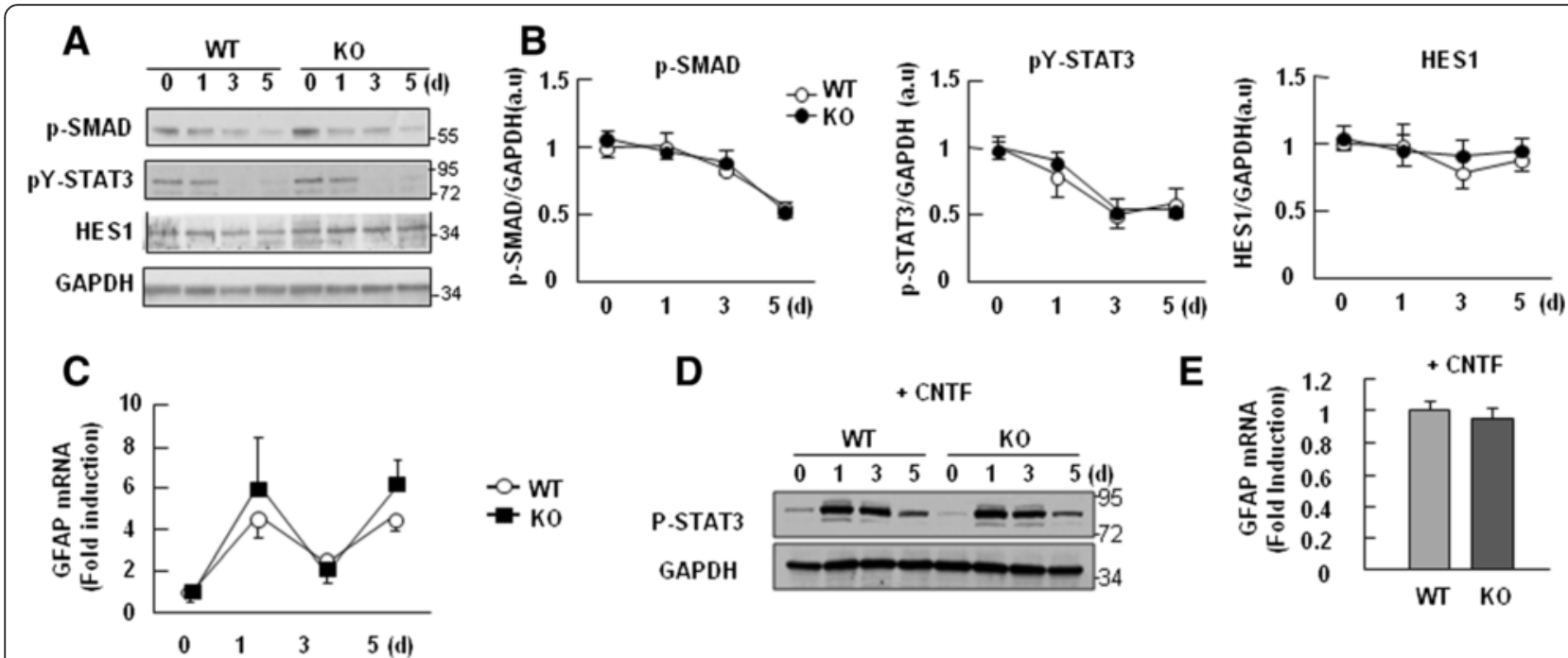

D

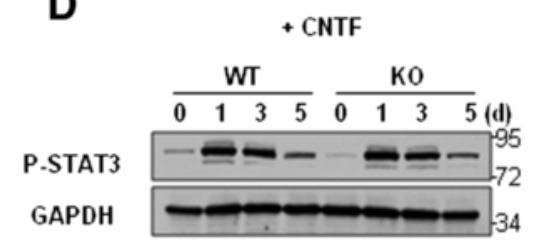

E

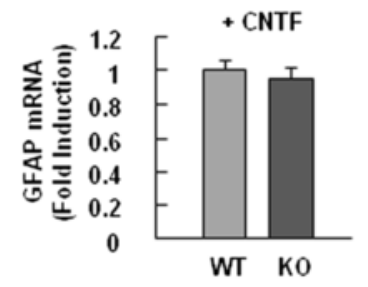

Fig. 4 PINK1 deficiency did not affect GFAP mRNA expression or the signaling pathways involved in astrocyte differentiation. a, b The levels of proteins known to be involved in astrocyte differentiation-related pathways (e.g., p-SMAD1/5/8, p-STAT3, and HES1) were measured by Western blotting (a). GAPDH was used as the loading control. The band intensities of p-SMAD1/5/8, p-STAT3, and HES1 were quantified in (b). c GFAP mRNA levels were measured in WT and PINK1-KO NSCs using Q-PCR on days $0,1,3$, and 5 of differentiation. $\mathbf{d}$, e WT and PINK1-KO NSCS were differentiated in the presence of $0.1 \mathrm{ng} / \mathrm{ml} \mathrm{CNTF}$ for 5 days. p-STAT3 levels were compared after the indicated durations of differentiation (d), and GFAP mRNA levels were examined on day 3 of differentiation (e). Values are means \pm SEM of at least four samples $(\mathbf{b}, \mathbf{c}, \mathbf{e})$

in the location and/or distribution of GFAP-positive astrocytes in the SVZ and/or SN.

In injured brain, SVZ-NSCs migrate toward injury sites and differentiate into astrocytes as well as neurons [45-49]. Astrocytes contribute to restoring disrupted extracellular fluid homeostasis and repairing the injured brain: astrocytes increase expression of glutamate and potassium transporters $[25,26]$, facilitate axon regeneration [50-52], constitute a part of the neurogenic niche [53-55], and affect neurogenesis [56, 57]. Accordingly, in ischemic brain, disruption of the differentiation of SVCNSCs to astrocytes induces abnormal astrogliosis, which results in an exaggerated microvascular hemorrhage [46]. Therefore, defects in astrogliogenesis and/or astrocyte functions can decrease neuronal support and impair the repair of injured brain, potentially leading to gradual neuronal death and accumulation of damage, which results in neurodegenerative diseases [31, 58-61].

Next arising question was how PINK1 decreases differentiation of PINK1-KO NSCs into GFAP-positive astrocytes. We excluded the possible involvement of cell death in the decreased differentiation of PINK1-KO NSCs into GFAP-positive astrocytes, as assessed by the amounts of cleaved PARP, cleaved caspase-3, and LDH release (Additional file 4: Figure S4). Additionally, PINK1 deficiency did not switch the balance of NSC differentiation from neurogenesis to gliogenesis, since the number of TUJ-1-positive cells did not increase (Fig. 3c, e). During differentiation of WT and PINK1-KO NSCs, mRNA levels of GFAP did not differ significantly (Fig. 4c), and the activation levels of the signaling pathways involved in gliogenesis, such as STAT3 [36-38], SMAD1/5/8 [39], and HES1 [40] were also little different (Fig. 4b). Furthermore, mitochondrial dysfunction that has been found in PINK1 deficient neurons and astrocytes $[2,7,44]$ was not detectable in PINK1-KO NSCs before and after the induction of differentiation (Fig. 5). Although mitochondrial dysfunction retarded proliferation of PINK1 deficient astrocytes [7], the proliferation of PINK1-KO NSCs may be normal based on their normal mitochondrial function (Fig. 2). These findings suggest that PINK1 may not be required for normal mitochondrial function in NSCs differentiation and/or that other genes may substitute for PINK1 in this case. It is also possible that PINK1-induced mitochondrial defects may accumulate in an age-dependent manner. Since GFAP mRNA expression was not reduced at PINK1-KO NSCs, we further examined the effect of proteasome inhibitors, MG132 and lactacystin, on GFAP expression (Additional file 5: Figure S5). Interestingly, these inhibitors had little effect, suggesting that PINK1 did not alter the protein stability of GFAP. Recently, it has been reported that several PD genes may regulate protein translation [62]. Therefore, further studies should be done to assess whether PINK1 may regulate GFAP expression at translation levels.

The importance of glia in the maintenance of brain function is beyond question, and their loss and/or abnormal function can contribute to neurodegeneration [63]. Our group and others have reported that mutations in several PD genes can alter the functions of astrocytes 


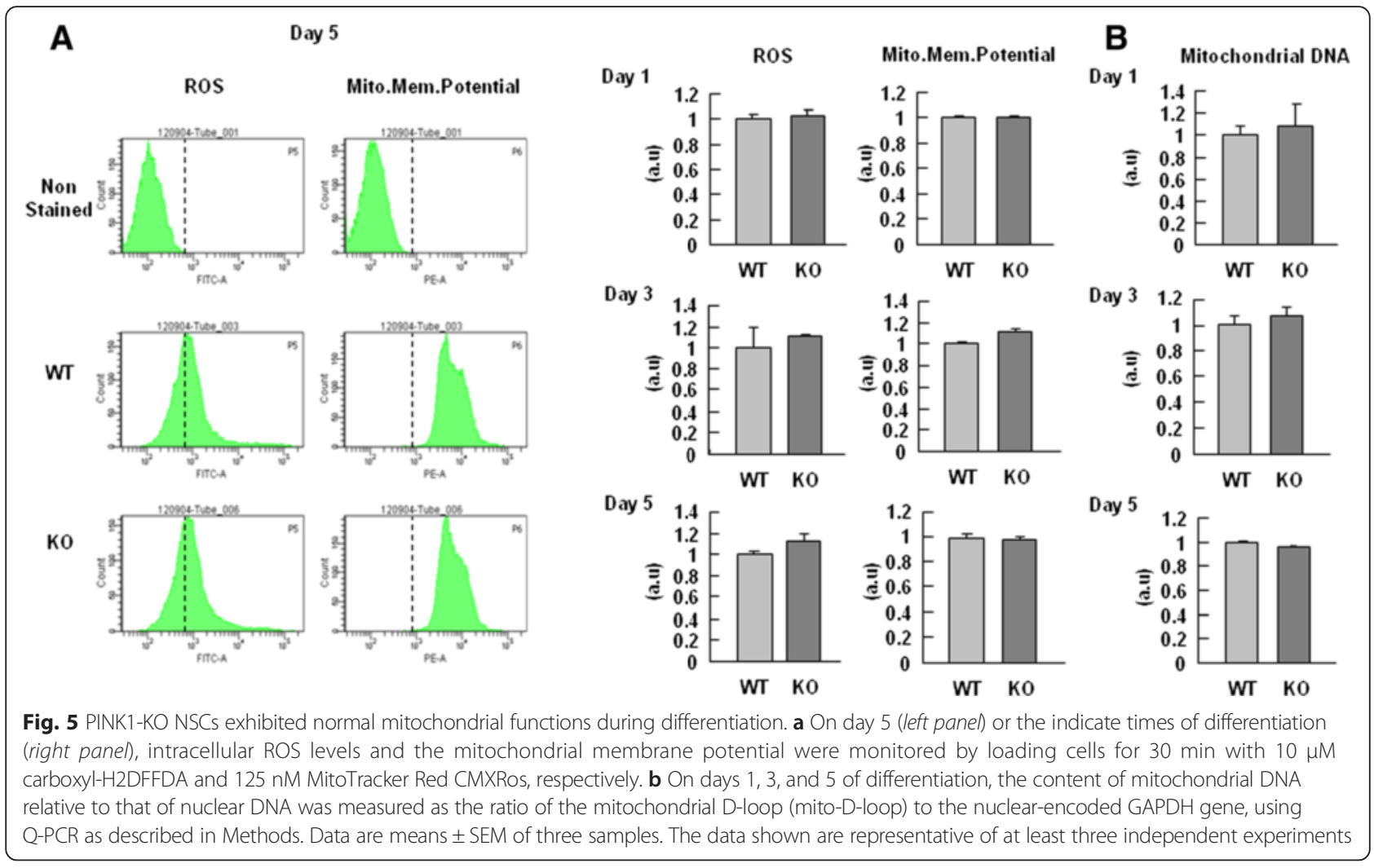

and other brain cells, including NSCs and microglia. For example, mutation of DJ-1 attenuates the neuroprotective functions of astrocytes [64]. Studies have shown that the inflammation and endocytosis of astrocytes and microglia can be regulated by DJ-1, PINK1, and LRRK2 [65-67], while the proliferation capacity of astrocytes is regulated by PINK1 [7], and LRRK2 mutation affects the viability of stem cells [68]. In this study, we found that Parkin similar to PINK1 changed its expression during development of the brain and NSC differentiation (Additional file 1: Figure S1, Additional file 3: Figure S3) although both Parkin and PINK1 in monkey are decreased or remain unchanged during aging [69]. Taken together, these lines of evidence suggest that PD does not affect only neurons, but rather is also a disease of other brain cells, including astrocytes and NSCs.

\section{Conclusion}

In conclusion, we herein provide the first evidence that PINK1 deficiency causes defects in the differentiation of NSCs to astrocytes and/or delay in GFAP expression and/or development of GFAP-expressing cells. Since astrocytes play critical roles in neuronal survival and the repair ininjured brain, insufficient astrocytic support due to PINK1 deficiency may cause neuronal death and/or abnormal tissue repair of the injured brain, accumulating damage and increasing the risk of PD. These possibilities imply that neurodegenerative diseases, including PD, could be diseases of astrocytes as well as neurons. Therefore, the functional regulation of non-neuronal cells should be a new target for the development of therapies for PD.

\section{Methods}

\section{Animals}

The PINK1-deficient mice were a generous gift from Dr. Xiaoxi Zhuang (Chicago University) and Dr. UJ Kang (Columbia University), and were as previously described [7]. All animal procedures were approved by the Ajou University School of Medicine Ethics Review Committee for Animal Research (Amc-119).

\section{Neurosphere culture and cell counting}

Embryonic neurospheres were cultured from the brains of embryonic day 13.5 (E13.5) mice, as previously described [70]. Briefly, forebrains were freed of meninges and gently triturated several times in culture medium using a flame-polished Pasteur pipette. Cells from a single brain were plated in a $100-\mathrm{mm}$ Petri dish and cultured in Dulbecco's modified Eagle's medium (DMEM)/ F12 medium (WelGene, Daegu, Korea) supplemented with N-2, B27 supplement (Gibco-Invitrogen, Carlsbad, CA, USA), $20 \mathrm{ng} / \mathrm{ml} \mathrm{EGF,} \mathrm{and} \mathrm{bFGF} \mathrm{(BD} \mathrm{Bioscience,}$ San Jose, CA, USA). EGF and bFGF were added every 


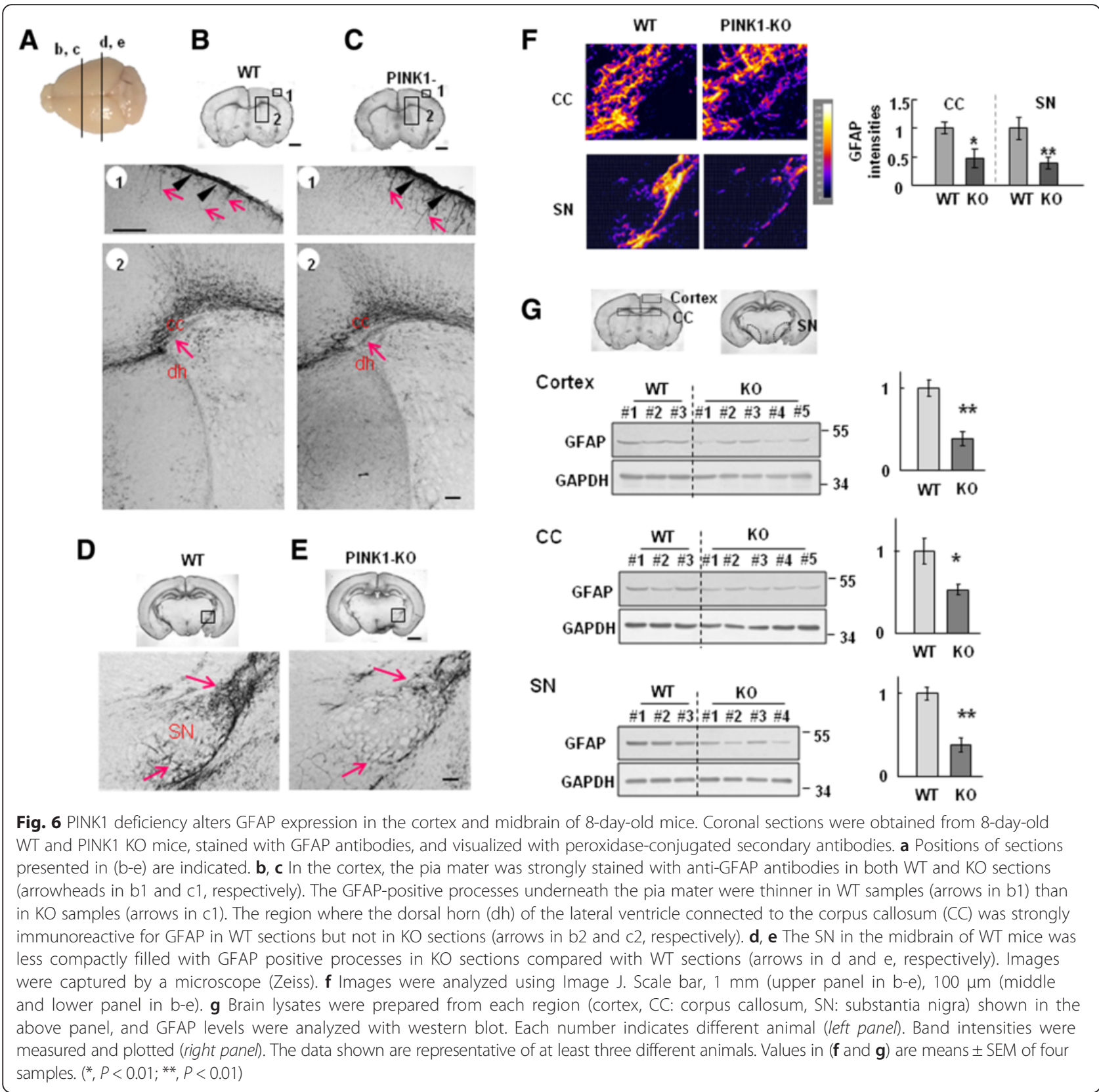

2 days. For serial neurosphere formation, primary neurospheres were collected, incubated with Accumax (Millipore), and dissociated. For differentiation, dissociated cells were seeded on plates coated with $0.2 \mathrm{mg} / \mathrm{ml}$ poly-L-ornithine and $1 \mu \mathrm{g} / \mathrm{ml}$ fibronectin (Sigma) in the absence of growth factors or in the presence of CNTF (BD Bioscience).

For proliferation assays, dissociated primary neurospheres $\left(2 \times 10^{4}\right.$ cell/well $)$ were seeded to a 96 -well plate and incubated in the presence of EGF and bFGF for 3 days, and the sizes and numbers of secondary neurospheres were analyzed using the TINA software (Raytest, Straubenhardt, Germany). For the cell counting and $\left[{ }^{3} \mathrm{H}\right]$-thymidine incorporation assays, dissociated primary neurospheres $\left(1 \times 10^{5} \mathrm{cell} /\right.$ well $)$ were seeded to a polyL-ornithine- and fibronectin-coated 24-well plate in the presence of growth factors (added daily to prevent NSC differentiation). For cell counting, on the indicated day, adherent NSCs were incubated with $\mathrm{Ca}^{2+} / \mathrm{Mg}^{2+}$-free HBSS for $20 \mathrm{~min}$, detached by pipetting, and counted. For the thymidine incorporation assay, $1 \mu \mathrm{Ci} / \mathrm{ml}\left[{ }^{3} \mathrm{H}\right]$ thymidine was added on day 1 of culture. After $24 \mathrm{~h}$, the adherent NSCs were washed three times with PBS and lysed with $0.1 \mathrm{~N} \mathrm{NaOH}$. Radioactivity was determined using a $\beta$-counter (Packard Instruments, Downers Grove, IL, USA). 


\section{Western blot analysis}

Cells and mouse brains were lysed on ice in RIPA buffer (50 mM Tris- $\mathrm{HCl}, \mathrm{pH}$ 7.4, 1 \% NP-40, $0.25 \% \mathrm{Na}$ deoxycholate, $150 \mathrm{mM} \mathrm{NaCl}, 1 \mathrm{mM} \mathrm{Na} \mathrm{VO}_{4}$, and $1 \mathrm{mM}$ $\mathrm{NaF}$ ) containing protease inhibitors $(2 \mathrm{mM}$ phenylmethylsulfonyl fluoride [PMSF], $10 \mu \mathrm{g} / \mathrm{ml}$ leupeptin, $10 \mu \mathrm{g} / \mathrm{ml}$ pepstatin, and $2 \mathrm{mM}$ EDTA) and a phosphatase inhibitor cocktail (GenDEPOT, Barker, TX, USA). Proteins were separated by SDS-PAGE, transferred to nitrocellulose membranes, and identified using specific antibodies. The antibodies for PINK1 (Cat. NO. 23707), MAP2, and MBP were obtained from Abcam (Cambridge, MA, USA); for Parkin, p-SMAD1/5/8, and p-STAT3 from Cell signaling technology (Danvers, MA, USA); for nestin and CNPase from Millipore (Bedford, MA, USA); for TUJ-1 from Covance (Berkeley, CA, USA); for GFAP from Sigma (Cat. No. G3893; St. Louis, MO, USA); and for HES1 and GAPDH from Santa Cruz Biotechnology (Santa Cruz, CA, USA). Membranes were incubated with peroxidase-conjugated secondary antibodies (Jackson Immuno Research, West Grove, PA, USA), and visualized with an enhanced chemiluminescence system (Daeil Lab Inc., Seoul, Korea).

\section{Q-PCR}

Total RNA was isolated using RNAzol B (iNtRON, Sungnam, Korea), and cDNA was prepared using Avian Myeloblastosis Virus reverse transcriptase (Promega, Madison, WI, USA) according to the manufacturer's instructions. The relevant mRNA levels were measured using a KAP SYBR FAST qPCR kit (Kapa Biosystems, Boston, MA, USA) and a RotoGene thermocycler (Corbett Research, Sydney, Australia). The primer pairs used in this study were synthesized by Integrated DNA Technologies (Coralville, IA, USA) and were as follows: PINK1, 5'-GC TTGCCAATCCCTTCTATG-3' (sense) and 5'-CTCTCGC TGGAGCAGTGAC-3' (antisense); GFAP, 5'-AGCTAGC CCTGGACATCGAGA-3'(sense) and 5'-GGTGAGCCTG TATTGGGACAA-3'(antisense); GAPDH (reference housekeeping gene), 5'-GCCTTCCGTGTTCCTACC-3' (sense) and 5'-CCTCAGTGTAGCCCAAGATG-3' (antisense). The cycle thresholds $(\mathrm{Ct})$ for the PINK1 and GFAP gene transcripts were normalized to the average $\mathrm{Ct}$ for GAPDH, and the relative quantitation of normalized transcript abundance was determined using the comparative $\mathrm{Ct}$ method $(\Delta \Delta \mathrm{Ct})$, as described by the manufacturer (Kapa Biosystems, Boston, MA, USA).

\section{Tissue preparation for immunostaining}

Mice were anesthetized and transcardially perfused with saline solution containing $0.5 \%$ sodium nitrate and heparin (10 Unit $/ \mathrm{ml})$, and then with $4 \%$ paraformaldehyde in $0.1 \mathrm{M}$ phosphate buffer (PB, $\mathrm{pH}$ 7.4). Brains were obtained and post-fixed overnight at $4{ }^{\circ} \mathrm{C}$ in $4 \%$ paraformaldehyde.
Fixed brains were stored at $4{ }^{\circ} \mathrm{C}$ in a $30 \%$ sucrose solution until they sank. Series of coronal sections $(30 \mu \mathrm{m})$ were obtained with a cryostat (Leica, Wetzlar, Germany), and used for immunohistochemistry.

\section{Immunostaining}

For 3, 3'-diaminobenzidine (DAB) staining, brain sections were rinsed three times with PBS, treated with $3 \%$ $\mathrm{H}_{2} \mathrm{O}_{2}$ for 5 min, and rinsed with PBS containing $0.2 \%$ Triton X-100 (PBST). Non-specific binding was blocked with $1 \%$ BSA in PBST. Sections were incubated overnight at room temperature with primary antibodies specific for GFAP (Neuromics, Minneapolis, MN, USA; Cat. No. RA22101). The sections were then rinsed with PBST, incubated with biotinylated secondary antibodies (Vector Laboratories, Burlingame, CA, USA), and visualized as described by the manufacturer (Vector Laboratories). Sections were mounted on gelatin-coated slides, and examined under bright field microscopy (Olympus Optical, BX51, Tokyo, Japan).

Cells were fixed with $4 \%$ paraformaldehyde at room temperature for $20 \mathrm{~min}$, washed with $\mathrm{PBS}$, and incubated with $1 \%$ BSA and $0.1 \%$ Triton X-100 in PBS for $30 \mathrm{~min}$. The cells were then incubated overnight with anti-GFAP, -TUJ-1, -MAP2, and -CNPase antibodies at $4{ }^{\circ} \mathrm{C}$, washed with PBS, and incubated with fluoresceinconjugated secondary antibodies (Invitrogen) for $2 \mathrm{~h}$. Finally, the cells were washed, mounted using a mounting medium containing 4', 6-diamidino-2-phenylindole (DAPI; Vector Laboratories), and examined under an Axiovert 200 M microscope (Carl Zeiss, Göttingen, Germany).

\section{Measurement of mitochondrial-membrane potential and intracellular reactive oxygen species}

NSCs were plated in 6-well plates $\left(1.5 \times 10^{6}\right.$ cells/well $)$. Mitochondrial membrane potential and intracellular reactive oxygen species (ROS) were monitored by loading cells for 30 min with $125 \mathrm{nM}$ MitoTracker Red CMXRos and $10 \mu \mathrm{M}$ carboxyl-H2DFFDA, respectively, as described previously [71]. Cells were washed twice with PBS and detached with Cellstripper TM (Media Tech, Inc., Manassas, VA, USA). Fluorescence intensities of detached cells were analyzed with a fluorescence-activated cell sorter (FACS; B-D FACS Systems, Sunnyvale, CA, USA).

\section{Measurement of mitochondrial DNA}

For assessment of the mitochondrial DNA copy number, genomic DNA was isolated using an Exgene Cell SV kit (GeneAll, Seoul, Korea), and the content of mitochondrial DNA relative to that of nuclear DNA was measured as the ratio of the mitochondrial D-loop (mito-D-loop) to the nuclear-encoded GAPDH gene, using Q-PCR. A RotoGene thermocycler (Corbett Research, Sydney, Australia) was used with a KAP SYBR FAST qPCR kit (Kapa 
Biosystems, Boston, MA, USA), and the following primer pairs: mito-D-loop, 5'- CCC AAG CAT ATA AGC TAG TAC-3' (sense) and 5'- ATA TAA GTC ATA TTT TGG GAA CTA C -3' (antisense); and GAPDH, 5'-GCCTTCC GTGTTCCTACC-3' (sense) and 5'-CCTCAGTGTAGCC CAAGATG-3' (antisense). The cycle threshold (Ct) for the mito-D-loop transcript was normalized to the average $\mathrm{Ct}$ for GAPDH in each reaction. Relative quantification of normalized transcript abundance was performed using the comparative $\mathrm{Ct}$ method $(\Delta \Delta \mathrm{Ct})$.

\section{Statistical analysis}

All data presented in this study are representative of at least three independent experiments. The statistical significance of differences between mean values of two groups was assessed by the Student's t-test. For comparisons of more than two groups, we used one-way ANOVA with Duncan's post-hoc test.

\section{Additional files}

Additional file 1: Figure S1. Parkin expression increased during brain development. Mouse brain lysates were collected at the indicated ages. The levels of Parkin were assayed by Western blotting. As PINK1, Parkin expression increased. (TIF 79 kb)

Additional file 2: Figure S2. Confirmation of absence of PINK1 protein in PINK1 KO mice. Cell lysates were prepared from NSCs on day 5 of differentiation. PINK1 expression was analyzed with Western-blot. (TIF $61 \mathrm{~kb}$ )

Additional file 3: Figure S3. Parkin expression increased during NSC differentiation. Cell lysates were collected on day of differentiation of NSCs. The levels of Parkin were assayed by Western blotting. As PINK1, Parkin expression increased. The data shown are representative of at least three independent experiments. (TIF $68 \mathrm{~kb}$ )

Additional file 4: Figure S4. PINK1 deficiency did not affect the viability of NSCs during differentiation. (a) The viability of WT and PINK1-KO NSCs was examined by Western-blotting of cleaved PARP and cleaved caspase-3 on day 5 of differentiation. GAPDH was used as the loading control. The band intensities of PARP and caspase-3 were quantified (right panel). (b) LDH release was measured with an LDH-Cytotoxicity Assay Kit (Biovision, Mountain View, CA, USA). Data are presented as the means \pm SEM of three samples. The data shown are representative of at least three independent experiments. (TIF $113 \mathrm{~kb}$ )

Additional file 5: Figure S5. Blocking of protein degradation does not increase GFAP protein levels in PINK1-KO NSCs. On day 4 of differentiation, PINK1-KO NSCs were treated with the indicated amounts of a proteasomal inhibitor (MG132) or a lysosomal inhibitor (lactacystin) for $24 \mathrm{~h}$, and GFAP levels were analyzed by Western blotting. The data shown are representative of at least three independent experiments. (TIF $64 \mathrm{~kb}$ )

\section{Abbreviation}

GFAP: Glial fibrillary acidic protein; PD: Parkinson's disease; PINK1: PTENinduced kinase 1.

\section{Competing interests}

The authors declare that they have no competing interests.

\section{Authors' contribution}

EJ conceived, supervised the project, and wrote the paper. IC designed, performed, and analyzed most of the experiments and wrote the paper. DC and HY performed animal experiments. JK and MC assisted neural stem cell culture. WS and SL supported neural stem cell culture, and JW, SP, and IJ contributed towrite the paper. All authors read and approved the final manuscript.

\section{Acknowledgements}

This work was supported by a grant (NRF-2014R1A2A2A01005947) funded by the Korean government (MEST) and a grant (NRF-2012R1A5A2048183) from KOSEF through the Chronic Inflammatory Disease Research Center (CIDRC) at Ajou University.

\section{Author details}

${ }^{1}$ Neuroscience Graduate Program Department of Biomedical Sciences, Ajou University School of Medicine, Suwon, Korea. ${ }^{2}$ Chronic Inflammatory Disease Research Center, Ajou University School of Medicine, Suwon, Korea. ${ }^{3}$ Department of Pharmacology, Ajou University School of Medicine san-5, Woncheon-dong, Youngtong-gu, Suwon, Kyunggi-do 442-721, Korea. ${ }^{4}$ Department of Biochemistry and Molecular Biology, College of Medicine, Hanyang University, Seoul, Korea. ${ }^{5}$ Department of Anatomy and Division of Brain Korea 21 Plus Biomedical Science, Korea University College of Medicine, Seoul 136-705, Korea. 'Department of Brain Science, Ajou University School of Medicine, Suwon, Korea. ${ }^{7}$ Brain Disease Research Center, Ajou University School of Medicine, Suwon, Korea.

Received: 31 July 2015 Accepted: 4 January 2016 Published online: 08 January 2016

\section{References}

1. Valente EM, Bentivoglio AR, Dixon PH, Ferraris A, lalongo T, Frontali M, et al. Localization of a novel locus for autosomal recessive early-onset parkinsonism, PARK6, on human chromosome 1p35-p36. Am J Hum Genet. 2001;68(4):895-900.

2. Valente EM, Abou-Sleiman PM, Caputo V, Muqit MM, Harvey K, Gispert S, et al. Hereditary early-onset Parkinson's disease caused by mutations in PINK1. Science. 2004:304(5674):1158-60. doi:10.1126/science.1096284.

3. Beilina A, Van Der Brug M, Ahmad R, Kesavapany S, Miller DW, Petsko GA, et al. Mutations in PTEN-induced putative kinase 1 associated with recessive parkinsonism have differential effects on protein stability. Proc Natl Acad Sci U S A. 2005:102(16):5703-8. doi:10.1073/pnas.0500617102.

4. Liu W, Vives-Bauza C, Acin-Perez R, Yamamoto A, Tan Y, Li Y, et al. PINK1 defect causes mitochondrial dysfunction, proteasomal deficit and alphasynuclein aggregation in cell culture models of Parkinson's disease. PLoS ONE. 2009;4(2):e4597. doi:10.1371/journal.pone.0004597.

5. $\operatorname{Sim} \mathrm{CH}$, Lio DS, Mok SS, Masters CL, Hill AF, Culvenor JG, et al. C-terminal truncation and Parkinson's disease-associated mutations down-regulate the protein serine/threonine kinase activity of PTEN-induced kinase-1. Hum Mol Genet. 2006;15(21):3251-62. doi:10.1093/hmg/ddl398.

6. Gandhi S, Wood-Kaczmar A, Yao Z, Plun-Favreau H, Deas E, Klupsch K, et al. PINK1-associated Parkinson's disease is caused by neuronal vulnerability to calcium-induced cell death. Mol Cell. 2009;33(5):627-38. doi:10.1016/j.molcel 2009.02.013.

7. Choi I, Kim J, Jeong HK, Kim B, Jou I, Park SM, et al. Pink1 deficiency attenuates astrocyte proliferation through mitochondrial dysfunction, reduced akt and increased p38 mapk activation, and downregulation of egfr. Glia. 2013;61(5):800-12. doi:10.1002/glia.22475.

8. Deng H, Jankovic J, Guo Y, Xie W, Le W. Small interfering RNA targeting the PINK1 induces apoptosis in dopaminergic cells SH-SY5Y. Biochem Biophys Res Commun. 2005;337(4):1133-8. doi:10.1016/j.bbrc.2005.09.178.

9. Haque ME, Thomas KJ, D'Souza C, Callaghan S, Kitada T, Slack RS, et al. Cytoplasmic Pink1 activity protects neurons from dopaminergic neurotoxin MPTP. Proc Natl Acad Sci U S A. 2008;105(5):1716-21. doi:10.1073/pnas. 0705363105.

10. Requejo-Aguilar R, Lopez-Fabuel I, Fernandez E, Martins LM, Almeida A, Bolanos JP. PINK1 deficiency sustains cell proliferation by reprogramming glucose metabolism through HIF1. Nat Commun. 2014;5:4514. doi:10.1038/ ncomms5514.

11. Lin W, Wadlington NL, Chen L, Zhuang X, Brorson JR, Kang UJ. Loss of PINK1 attenuates HIF-1alpha induction by preventing 4E-BP1-dependent switch in protein translation under hypoxia. J Neurosci. 2014;34(8):3079-89. doi:10.1523/JNEUROSCI.2286-13.2014.

12. Kim J, Byun JW, Choi I, Kim B, Jeong HK, Jou I, et al. PINK1 Deficiency Enhances Inflammatory Cytokine Release from Acutely Prepared Brain Slices. Exp Neurobiol. 2013;22(1):38-44. doi:10.5607/en.2013.22.1.38. 
13. Dagda RK, Pien I, Wang R, Zhu J, Wang KZ, Callio J, et al. Beyond the mitochondrion: cytosolic PINK1 remodels dendrites through protein kinase A. J Neurochem. 2014;128(6):864-77. doi:10.1111/jnc.12494.

14. Ullian EM, Sapperstein SK, Christopherson KS, Barres BA. Control of synapse number by glia. Science. 2001;291(5504):657-61. doi:10.1126/science.291.5504.657.

15. Beattie EC, Stellwagen D, Morishita W, Bresnahan JC, Ha BK, Von Zastrow M, et al. Control of synaptic strength by glial TNFalpha. Science. 2002; 295(5563):2282-5. doi:10.1126/science.1067859.

16. Gage FH, Olejniczak P, Armstrong DM. Astrocytes are important for sprouting in the septohippocampal circuit. Exp Neurol. 1988;102(1):2-13.

17. Sakatani S, Seto-Ohshima A, Itohara S, Hirase H. Impact of S100B on local field potential patterns in anesthetized and kainic acid-induced seizure conditions in vivo. Eur J Neurosci. 2007;25(4):1144-54. doi:10.1111/j.1460-9568.2007.05337.x.

18. Brown AM, Ransom BR. Astrocyte glycogen and brain energy metabolism. Glia. 2007;55(12):1263-71. doi:10.1002/glia.20557.

19. Chih CP, Roberts Jr EL. Energy substrates for neurons during neural activity: a critical review of the astrocyte-neuron lactate shuttle hypothesis. J Cereb Blood Flow Metab. 2003;23(11):1263-81. doi:10.1097/01.WCB.0000081369.51727.6F.

20. Pellerin L, Bouzier-Sore AK, Aubert A, Serres S, Merle M, Costalat R, et al. Activity-dependent regulation of energy metabolism by astrocytes: an update. Glia. 2007;55(12):1251-62. doi:10.1002/glia.20528.

21. Anderson CM, Swanson RA. Astrocyte glutamate transport: review of properties, regulation, and physiological functions. Glia. 2000;32(1):1-14.

22. Simard $M$, Nedergaard $M$. The neurobiology of glia in the context of water and ion homeostasis. Neuroscience. 2004;129(4):877-96. doi:10.1016/j. neuroscience.2004.09.053.

23. Wilson JX. Antioxidant defense of the brain: a role for astrocytes. Can J Physiol Pharmacol. 1997;75(10-11):1149-63.

24. Jeong HK, Jou I, Joe EH. Absence of Delayed Neuronal Death in ATP-Injected Brain: Possible Roles of Astrogliosis. Exp Neurobiol. 2013;22(4):308-14. doi:10.5607/en.2013.22.4.308.

25. Jeong HK, Ji KM, Kim J, Jou I, Joe EH. Repair of astrocytes, blood vessels, and myelin in the injured brain: possible roles of blood monocytes. Mol Brain. 2013;6:28. doi:10.1186/1756-6606-6-28

26. Jeong HK, Ji KM, Min KJ, Choi I, Choi DJ, Jou I, et al. Astrogliosis is a possible player in preventing delayed neuronal death. Mol Cells. 2014;37(4):345-55. 10.14348/molcells.2014.0046.

27. Kim JH, Min KJ, Seol W, Jou I, Joe EH. Astrocytes in injury states rapidly produce anti-inflammatory factors and attenuate microglial inflammatory responses. J Neurochem. 2010;115(5):1161-71. doi:10.1111/j.1471-4159.2010.07004.x.

28. Min KJ, Yang MS, Kim SU, Jou I, Joe EH. Astrocytes induce hemeoxygenase-1 expression in microglia: a feasible mechanism for preventing excessive brain inflammation. J Neurosci. 2006;26(6):1880-7. doi:10.1523/ JNEUROSCI.3696-05.2006.

29. Gonzalez-Perez O, Quinones-Hinojosa A. Astrocytes as neural stem cells in the adult brain. J Stem Cells. 2012;7(3):181-8. jsc.2012.7.3.181.

30. Magnusson JP, Goritz C, Tatarishvili J, Dias DO, Smith EM, Lindvall O, et al. A latent neurogenic program in astrocytes regulated by Notch signaling in the mouse. Science. 2014;346(6206):237-41. doi:10.1126/science.346.6206.237.

31. Armstrong RJ, Barker RA. Neurodegeneration: a failure of neuroregeneration? Lancet. 2001;358(9288):1174-6. doi:10.1016/S0140-6736(01)06260-2.

32. Miller FD, Gauthier AS. Timing is everything: making neurons versus glia in the developing cortex. Neuron. 2007;54(3):357-69. doi:10.1016/..neuron.2007.04.019.

33. Lucking CB, Durr A, Bonifati V, Vaughan J, De Michele G, Gasser T, et al. Association between early-onset Parkinson's disease and mutations in the parkin gene. N Engl J Med. 2000;342(21):1560-7. doi:10.1056/ NEJM200005253422103.

34. Xiong H, Wang D, Chen L, Choo YS, Ma H, Tang C, et al. Parkin, PINK1, and DJ-1 form a ubiquitin E3 ligase complex promoting unfolded protein degradation. J Clin Invest. 2009;119(3):650-60. doi:10.1172/JCl37617.

35. Rajan P, McKay RD. Multiple routes to astrocytic differentiation in the CNS. J Neurosci. 1998;18(10):3620-9.

36. Bonni A, Sun Y, Nadal-Vicens M, Bhatt A, Frank DA, Rozovsky I, et al. Regulation of gliogenesis in the central nervous system by the JAK-STAT signaling pathway. Science. 1997;278(5337):477-83

37. He F, Ge W, Martinowich K, Becker-Catania S, Coskun V, Zhu W, et al. A positive autoregulatory loop of Jak-STAT signaling controls the onset of astrogliogenesis. Nat Neurosci. 2005;8(5):616-25. doi:10.1038/nn1440.

38. Nakashima K, Yanagisawa M, Arakawa H, Kimura N, Hisatsune T, Kawabata $\mathrm{M}$, et al. Synergistic signaling in fetal brain by STAT3-Smad1 complex bridged by p300. Science. 1999;284(5413):479-82.
39. Nakashima K, Takizawa T, Ochiai W, Yanagisawa M, Hisatsune T, Nakafuku M, et al. BMP2-mediated alteration in the developmental pathway of fetal mouse brain cells from neurogenesis to astrocytogenesis. Proc Natl Acad Sci U S A. 2001;98(10):5868-73. doi:10.1073/pnas.101109698.

40. Kamakura S, Oishi K, Yoshimatsu T, Nakafuku M, Masuyama N, Gotoh Y. Hes binding to STAT3 mediates crosstalk between Notch and JAK-STAT signalling. Nat Cell Biol. 2004;6(6):547-54. doi:10.1038/ncb1138.

41. Pera EM, Ikeda A, Eivers E, De Robertis EM. Integration of IGF, FGF, and anti-BMP signals via Smad1 phosphorylation in neural induction. Genes Dev. 2003;17(24):3023-8. doi:10.1101/gad.1153603.

42. Zhong Z, Wen Z, Darnell Jr JE. Stat3: a STAT family member activated by tyrosine phosphorylation in response to epidermal growth factor and interleukin-6. Science. 1994;264(5155):95-8.

43. Jouve C, Palmeirim I, Henrique D, Beckers J, Gossler A, Ish-Horowicz D, et al. Notch signalling is required for cyclic expression of the hairy-like gene HES1 in the presomitic mesoderm. Development. 2000;127(7):1421-9.

44. Silvestri L, Caputo V, Bellacchio E, Atorino L, Dallapiccola B, Valente EM, et al. Mitochondrial import and enzymatic activity of PINK1 mutants associated to recessive parkinsonism. Hum Mol Genet. 2005;14(22):3477-92. doi:10.1093/hmg/ddi377.

45. Arvidsson A, Collin T, Kirik D, Kokaia Z, Lindvall O. Neuronal replacement from endogenous precursors in the adult brain after stroke. Nat Med. 2002;8(9):963-70. doi:10.1038/nm747.

46. Benner EJ, Luciano D, Jo R, Abdi K, Paez-Gonzalez P, Sheng H, et al. Protective astrogenesis from the SVZ niche after injury is controlled by Notch modulator Thbs4. Nature. 2013;497(7449):369-73. doi:10.1038/nature12069.

47. Faiz M, Acarin L, Villapol S, Schulz S, Castellano B, Gonzalez B. Substantial migration of SVZ cells to the cortex results in the generation of new neurons in the excitotoxically damaged immature rat brain. Mol Cell Neurosci. 2008;38(2):170-82. doi:10.1016/j.mcn.2008.02.002.

48. Yamashita T, Ninomiya M, Hernandez Acosta P, Garcia-Verdugo JM, Sunabori T, Sakaguchi $M$, et al. Subventricular zone-derived neuroblasts migrate and differentiate into mature neurons in the post-stroke adult striatum. J Neurosci. 2006;26(24):6627-36. doi:10.1523/JNEUROSCI.0149-06.2006.

49. Zhang RL, Zhang ZG, Zhang L, Chopp M. Proliferation and differentiation of progenitor cells in the cortex and the subventricular zone in the adult rat after focal cerebral ischemia. Neuroscience. 2001;105(1):33-41.

50. Costa S, Planchenault T, Charriere-Bertrand C, Mouchel Y, Fages C, Juliano S, et al. Astroglial permissivity for neuritic outgrowth in neuron-astrocyte cocultures depends on regulation of laminin bioavailability. Glia. 2002;37(2):105-13.

51. Tom VJ, Doller CM, Malouf AT, Silver J. Astrocyte-associated fibronectin is critical for axonal regeneration in adult white matter. J Neurosci. 2004; 24(42):9282-90. doi:10.1523/JNEUROSCI.2120-04.2004.

52. White RE, Jakeman LB. Don't fence me in: harnessing the beneficial roles of astrocytes for spinal cord repair. Restor Neurol Neurosci. 2008;26(2-3):197-214.

53. Shimada IS, Peterson BM, Spees JL. Isolation of locally derived stem/ progenitor cells from the peri-infarct area that do not migrate from the lateral ventricle after cortical stroke. Stroke. 2010;41(9):e552-60. doi:10.1161/ STROKEAHA.110.589010.

54. Castelo-Branco G, Sousa KM, Bryja V, Pinto L, Wagner J, Arenas E. Ventral midbrain glia express region-specific transcription factors and regulate dopaminergic neurogenesis through Wnt-5a secretion. Mol Cell Neurosci. 2006:31(2):251-62. doi:10.1016/j.mcn.2005.09.014

55. Wagner J, Akerud P, Castro DS, Holm PC, Canals JM, Snyder EY, et al. Induction of a midbrain dopaminergic phenotype in Nurr1-overexpressing neural stem cells by type 1 astrocytes. Nat Biotechnol. 1999;17(7):653-9. doi:10.1038/10862.

56. Lim DA, Alvarez-Buylla A. Interaction between astrocytes and adult subventricular zone precursors stimulates neurogenesis. Proc Natl Acad Sci U S A. 1999;96(13):7526-31.

57. Episcopo FL, Tirolo C, Testa N, Caniglia S, Morale MC, Marchetti B. Reactive astrocytes are key players in nigrostriatal dopaminergic neurorepair in the MPTP mouse model of Parkinson's disease: focus on endogenous neurorestoration. Curr Aging Sci. 2013;6(1):45-55.

58. O'Keeffe GC, Tyers P, Aarsland D, Dalley JW, Barker RA, Caldwell MA. Dopamine-induced proliferation of adult neural precursor cells in the mammalian subventricular zone is mediated through EGF. Proc Natl Acad Sci U S A. 2009;106(21):8754-9.

59. Maragakis NJ, Rothstein JD. Mechanisms of Disease: astrocytes in neurodegenerative disease. Nat Clin Pract Neurol. 2006;2(12):679-89. doi:10.1038/ncpneuro0355. 
60. Rossi D, Volterra A. Astrocytic dysfunction: insights on the role in neurodegeneration. Brain Res Bull. 2009;80(4-5):224-32. doi:10.1016/j. brainresbull.2009.07.012.

61. Winner B, Kohl Z, Gage FH. Neurodegenerative disease and adult neurogenesis. Eur J Neurosci. 2011;33(6):1139-51. doi:10.1111/j.1460-9568. 2011.07613.x.

62. Taymans JM, Nkiliza A, Chartier-Harlin MC. Deregulation of protein translation control, a potential game-changing hypothesis for Parkinson's disease pathogenesis. Trends Mol Med. 2015;21(8):466-72. doi:10.1016/j. molmed.2015.05.004

63. Graeber MB, Streit WJ. Microglia: biology and pathology. Acta Neuropathol. 2010;119(1):89-105. doi:10.1007/s00401-009-0622-0.

64. Mullett SJ, Hinkle DA. DJ-1 knock-down in astrocytes impairs astrocytemediated neuroprotection against rotenone. Neurobiol Dis. 2009;33(1):28-36. doi:10.1016/..nbd.2008.09.013

65. Kim B, Yang MS, Choi D, Kim JH, Kim HS, Seol W, et al. Impaired inflammatory responses in murine Lrrk2-knockdown brain microglia. PLoS ONE. 2012;7(4):e34693. doi:10.1371/journal.pone.0034693.

66. Kim KS, Kim JS, Park JY, Suh YH, Jou I, Joe EH, et al. DJ-1 Associates with lipid rafts by palmitoylation and regulates lipid rafts-dependent endocytosis in astrocytes. Hum Mol Genet. 2013. doi:10.1093/hmg/ddt332.

67. Kim JH, Choi DJ, Jeong HK, Kim J, Kim DW, Choi SY, et al. DJ-1 facilitates the interaction between STAT1 and its phosphatase, SHP-1, in brain microglia and astrocytes: A novel anti-inflammatory function of DJ-1. Neurobiol Dis. 2013. doi:10.1016/j.nbd.2013.08.007.

68. Liu GH, Qu J, Suzuki K, Nivet E, Li M, Montserrat N, et al. Progressive degeneration of human neural stem cells caused by pathogenic LRRK2. Nature. 2012:491 (7425):603-7. doi:10.1038/nature11557.

69. Yang W, Wang G, Wang CE, Guo X, Yin P, Gao J, et al. Mutant alpha-synuclein causes age-dependent neuropathology in monkey brain. J Neurosci. 2015. 35(21):8345-58.

70. Kim YH, Chung Jl, Woo HG, Jung YS, Lee SH, Moon CH, et al. Differential regulation of proliferation and differentiation in neural precursor cells by the Jak pathway. Stem Cells. 2010;28(10):1816-28. doi:10.1002/stem.511.

71. Zhou R, Yazdi AS, Menu P, Tschopp J. A role for mitochondria in NLRP3 inflammasome activation. Nature. 2011;469(7329):221-5. doi:10.1038/ nature09663.

\section{Submit your next manuscript to BioMed Central and we will help you at every step:}

- We accept pre-submission inquiries

- Our selector tool helps you to find the most relevant journal

- We provide round the clock customer support

- Convenient online submission

- Thorough peer review

- Inclusion in PubMed and all major indexing services

- Maximum visibility for your research

Submit your manuscript at www.biomedcentral.com/submit

) Biomed Central 